\title{
Effects of Being Imitated on Motor Responses Evoked by Pain Observation: Exerting Control Determines Action Tendencies When Perceiving Pain in Others
}

\author{
Lize De Coster, ${ }^{1}$ Michael Andres, ${ }^{1,2}$ and Marcel Brass ${ }^{1,3}$ \\ ${ }^{1}$ Faculty of Psychology, Department of Experimental Psychology, Ghent University, B-9000 Ghent, Belgium, ${ }^{2}$ Institut de reserche en sciences \\ psychologiques, Université catholique de Louvain, B-1348 Louvain-la-Neuve, Belgium, and ${ }^{3}$ Radboud University NL-6525 HP Nijmegen, The Netherlands
}

\begin{abstract}
Brain-imaging research has shown that experiencing pain oneself and perceiving pain in others lead to a similar pattern of activation, suggesting that the latter is based on internal simulation of the observed pain. Further evidence for this idea stems from transcranial magnetic stimulation measuring corticospinal excitability (CSE). It has been demonstrated that our motor cortex is involved whenever we observe another person receiving painful stimulation to the hand (Avenanti et al., 2005). However, both decreases and increases of CSE have been described during pain observation, so the exact nature of these CSE changes has remained unclear so far. In the present study, we hypothesized that CSE changes are determined by the control that the observer has over the hand that receives painful stimulation. To test this hypothesis, we manipulated the control over the observed hand using a paradigm in which participants' movements are being imitated by a hand on screen - giving them full control over the hand - or not. Consistent with previous results, we evidenced a decrease in CSE when participants experienced no control over the hand that received painful stimulation. In contrast, inducing control resulted in an increase in CSE. We conclude that exerting control over the observed hand leads to a completely altered action tendency. Whereas an anesthetic response is typically observed in the absence of control, increasing control induces motor facilitation reminiscent of preparation of an avoidance response.
\end{abstract}

Key words: empathy for pain; imitation; motor TMS; sense of control; social cognition

\section{Introduction}

Since the study of Singer et al. (2004), it has been shown repeatedly that the observation of pain in a model results in pain-related brain activation in the observer (for review, see Lamm et al., 2011). More recently, transcranial magnetic stimulation (TMS) studies have investigated how our motor system responds when perceiving pain in others. Interestingly, these studies have shown that the observation of painful stimulation delivered to the hand of a human model induces a decrease in corticospinal excitability (CSE) in the hand of the observer (Avenanti et al., 2005; Avenanti et al., 2006). It has been argued that this inhibitory effect is similar to what happens on the motor level when experiencing pain oneself (Le Pera et al., 2001; Farina et al., 2003; Urban et al., 2004). However, recent findings indicate that the decrease in CSE observed while perceiving pain in others is not always found. It

Received Nov. 29, 2013; revised March 25, 2014; accepted April 12, 2014

Author contributions: L.D.C., M.A., and M.B. designed research; L.D.C. performed research; L.D.C. analyzed data; L.D.C., M.A., and M.B. wrote the paper.

This work was supported by Research Foundation Flanders (Grant FW010/ASP/321 to L.D.C.). M.A. is a Research Associate at the Fund for Scientific Research, Fonds National de la Recherche Scientifique (Belgium). We thank Patrick Santens for help in participant screening.

The authors declare no competing financial interests.

Correspondence should be addressed to Lize De Coster, Faculty of Psychology, Department of Experimental Psychology, Ghent University, Henri Dunantlaan 2, B-9000 Ghent, Belgium. E-mail: Lize.DeCoster@UGent.be.

DOI:10.1523/JNEUROSCI.5044-13.2014

Copyright $\odot 2014$ the authors $\quad 0270-6474 / 14 / 346952-06 \$ 15.00 / 0$ has been shown that this inhibition is reduced in individuals with high levels of trait-personal distress (Avenanti et al., 2009). Furthermore, Fitzgibbon et al. (2012) have shown that pain synesthetes (i.e., individuals who experience actual pain when observing injury to another) show a significant increase in CSE while observing pain in others. These discrepant results raise questions about the factors that determine the nature of CSE changes induced by pain observation. A potential hypothesis is that, in experiments demonstrating an anesthetic motor inhibition after painful stimulation, participants were unable to avoid the pain or predict the exact timing of painful stimulation (Le Pera et al., 2001; Urban et al., 2004). Similarly, in experiments in which participants perceive pain in others, the decrease in CSE is typically associated with an absence of control over the hand in pain. Conversely, high levels of personal distress or synesthetic sensations may enhance the feeling that pain is inflicted on one's own hand and activate motor control processes, resulting in increased CSE as a reflection of planning an avoidance reaction to the observed pain. In the present study, we tested the original hypothesis that the nature of the CSE changes evoked by perceiving others' hand receiving painful stimulation is determined by our ability to exert control over this hand.

We recently manipulated the sense of control participants had over an observed hand in pain using a well established imitation paradigm (De Coster et al., 2013). In an imitative 
condition, a hand on screen imitated participants, giving them perfect control over this hand. In a nonimitative condition, the hand was performing nonmatching movements. We showed that affective reactions to perceiving painful stimulation in others were enhanced after being imitated by the other person and that this enhancement was related to an increase in control. In the present study, this imitation paradigm allowed us to investigate whether inducing control over the hand on the screen determines whether perceiving pain in this hand will lead to increased or decreased CSE.

\section{Materials and Methods}

Participants. Twenty-five healthy young adult men (mean age $=22.44$ years, $S D=2.03$ ) participated in the study in exchange for 40 Euros and provided written consent beforehand. Participants had no history of neurological or psychiatric disorders, had normal or corrected-to normal vision, and were negative for the risk factors associated with TMS (Rossi et al., 2009). The procedures were noninvasive and were performed in accordance with the ethical standards laid down in the 1964 Declaration of Helsinki. The study was granted ethical approval by the Medical Ethical Review Board of Ghent University Hospital.

Experimental design. Blocks of trials consisted of two phases: an action phase in which movements of the subjects were imitated (exertingcontrol block) or not (not-exerting-control block) and a pain perception phase that immediately followed the action phase. In the pain perception phase, one of the following 10 pain videos was presented: "bore goes into the back of the hand," 'hammer is smacked on the back of the hand," "hot iron is pressed on the back of the hand," "knife cuts the back of the hand," "nail is knocked into the back of the hand with a hammer," "nail of the ring finger is pulled out of the hand," "paper makes a paper cut in the back of the hand," "pinchers pinch the back of the hand," "sandpaper is rubbed over the back of the hand," or "stapler puts a staple into the back of the hand." Alternatively, a neutral video was shown in which a still hand appeared on screen, serving as a baseline for the pain videos. Each pain video was combined two times with both an exerting-control and not-exerting-control block, whereas the neutral video was combined 20 times with each block to ensure that an equal number of pain and neutral videos were presented. Therefore, the experiment consisted of 80 trials. The association of the different pain/neutral videos with the different block conditions was completely randomized across participants.

Stimuli and apparatus. Stimulus material consisted of three types of $720 \times 576$ video clips created by professionals: a hand in a resting position, simple finger movements (for the action phase of the task), and pain videos showing a hand receiving pain stimulation (for the pain videos in the pain perception phase).

During the action phase of the experimental task, participants performed simple finger movements of the index, middle, ring, or little finger. These finger movements were recorded with a custom-built response device using light sensors. This device allowed us to use fingerlifting movements of participants as triggers for the presentation of the appropriate finger movement video. Temporal resolution was optimized (see Procedure) so that participants immediately viewed a videotaped finger movement on screen after initiating a finger movement with their own hand. For example, in an exerting-control block, the lifting of an index finger resulted in the presentation of the index finger lifting video, whereas the middle, ring, or little finger lifting video was shown in a not-exerting-control block. All finger movement clips had a total duration of $2000 \mathrm{~ms}$.

The perception phase of the experimental task consisted of the presentation of one of 10 pain videos in which painful stimulation was applied to the hand on screen or a resting state video in which the right hand was displayed palm down with fingers slightly spread. The position of the videotaped hand matched the position of the participants' right hand on the response box. All videos had a total duration of $8000 \mathrm{~ms}$. The resting state video served as a neutral/baseline video for the pain videos (Avenanti et al., 2009). Practical constraints (including timing of the experiment) detained us from using additional control conditions in which hands are innocuously touched by similar objects. Although several stud- ies have shown that CSE is modulated by observation of pain, but not of touch stimuli (Avenanti et al., 2005, Avenanti et al., 2010), we cannot exclude the possibility that our modulations are not specific for pain and can be extended to any hand-object interaction.

Procedure. Participants were seated in front of a standard computer screen at arm length and asked to place the four fingers of their right hand on a custom-made response box. Display of stimulus material and recording of responses were conducted with Presentation software (Neurobehavioral Systems). As soon as the videotaped right hand appeared on screen (resting state video), subjects were instructed to voluntarily move a randomly chosen finger that was placed on the response box. Immediately after movement of one of the subjects' fingers (delay $=0 \mathrm{~ms}$, estimate of intrinsic delay of computer/software $=66.93 \mathrm{~ms}$ ), a video was shown in which the hand on screen performed the same or a different movement for exerting-control and not-exerting-control blocks, respectively. After a random number between 10 and 15 of such movements (all imitative or all nonimitative), one of the pain videos or the neutral video was presented immediately. After a pain video, participants had to rate the behavioral statement "I felt pain on my own hand when I saw the hand on screen receiving painful stimulation" on a scale from -5 to +5 . During the pain videos, a TMS pulse was applied at the exact time when the painful tool contacted the skin surface. During the neutral video, the TMS pulse was delivered at $2900 \mathrm{~ms}$, corresponding to the average of the TMS pulse onset across all pain videos.

Before the start of the experiment, participants' TMS motor threshold was measured as described in the TMS and Electromyography section below. Afterward, they performed two practice blocks (both an exertingcontrol and a not-exerting-control block) in which a pain video was shown that was not used during the experimental phase and no TMS pulse was applied. During these practice blocks, it was verified whether participants understood all aspects of the experimental procedure.

Finally, at the end of the experiment, participants filled in the Interpersonal Reactivity Index (IRI; Davis, 1980; for Dutch translation, see De Corte et al., 2007), which is used as a measure of trait empathy. This questionnaire consists of 28 items that have to be rated on a five-point Likert scale and can be divided into four subscales: (1) Perspective Taking (PT, the tendency to spontaneously imagine and assume the cognitive perspective of another person); (2) Empathic Concern (EC, the tendency to feel sympathy and compassion for others in need); (3) Fantasy (FS, the tendency to project oneself into the place of fictional characters in books and videos); and (4) Personal Distress (PD, the extent to which an individual feels distress as a result of witnessing another's emotional distress). Cronbach's $\alpha$ in the current study for PT was 0.83 , for EC 0.74 , for FS 0.79 , and for PD 0.80 .

TMS and electromyography. Single-pulse TMS was delivered through a biphasic magnetic stimulator (Rapid ${ }^{2}$; Magstim) connected to a polyurethane-coated figure- 8 coil ( $5.4 \mathrm{~cm}$ inner diameter windings). The coil was held tangentially over the left hand motor area, with the handle pointing backward and forming an angle of $45^{\circ}$ with the sagittal plane. Participants wore earplugs to attenuate the coil noise. Electromyographical (EMG) activity was recorded with the ActiveTwo system (BioSemi). Sintered $11 \times 17 \mathrm{~mm}$ active $\mathrm{Ag}-\mathrm{AgCl}$ electrodes were placed over the right first dorsal interosseus (FDI) muscle and the right brachioradialis muscle (BR) in a belly-tendon arrangement. The FDI contributes to flex or abduct the index away from the middle finger, whereas the main action of the BR is to flex the forearm at the elbow. These muscles were chosen because they are involved, respectively, in finger and hand retraction, two reactions commonly observed in response to painful stimuli as used in our study. The hot spot in the hand motor area was established by locating a stimulation site where TMS elicited motor evoked potentials (MEPs) in the two muscles. TMS intensity was set at $110 \%$ of the resting motor threshold; that is, the minimum intensity to induce an MEP $\geq 50$ $\mu \mathrm{V}$ peak to peak in both muscles with $50 \%$ probability. In 14 of 25 participants, the TMS parameters were defined according to the FDI only because it was not possible to elicit MEPs in both muscles from the same stimulation site. The data collected from the BR in other participants were excluded from further analyses because the number of trials in which an MEP was observed during the experiment was too small. Average intensity $( \pm S D)$ was $71.25 \pm 16.98 \%$ of the maximal stimu- 
lator output. EMG signal was amplified (internal gain scaling), digitized at $2 \mathrm{kHz}$, highpass filtered at $3 \mathrm{~Hz}$, and stored on a PC for offline analysis.

Data analyses. Trials were excluded when the root mean square (RMS) of the background EMG signal recorded in the FDI $500 \mathrm{~ms}$ before TMS was $>50 \mu \mathrm{V}$. For each subject, the top and bottom $5 \%$ of MEPs were trimmed and the peak-to-peak amplitude of the remaining MEPs was computed using MATLAB. For both control conditions separately, the MEPs in each pain condition were expressed as a percentage of change with respect to its corresponding baseline as follows: $100 *$ (pain neutral)/neutral. The baseline conditions did not differ significantly from each other $\left(t_{(24)}=\right.$ $1.34, p=0.20)$.

Planned comparisons between exerting control and not exerting control over the observed hand were performed for behavioral and TMS data using paired $t$ tests. For the latter, additional analyses were performed to rule out that the effects described in the Results section were due to differences in background EMG activity. These analyses showed that our manipulation did not influence the RMS of the EMG signal recorded from the FDI during a $500 \mathrm{~ms}$ delay before the TMS (all $p>0.20$ ).

Pearson correlations were computed between the average ratings on each subscale of the IRI and the percentage of change in MEP amplitude in the exerting-control and notexerting-control conditions. One outlier participant was identified using Cook's distance and was subsequently removed from the correlation analysis.

\section{Results}

\section{Subjective reports}

Consistent with previous results (De Coster et al., 2013), a paired $t$ test revealed that scores were significantly higher in the exerting-control compared with the not-exerting-control condition $\left(t_{(24)}=2.31, p<0.05, d=\right.$ 0.15; Fig. 1).

\section{TMS data}

Planned comparisons of the percentage of change in MEP amplitude in the FDI showed a significant difference between the exerting-control and not-exerting-control condition $\left(_{(24)}=\right.$ $3.44, p<0.01, d=1.87)$. As shown in Figure 2, MEP amplitude decreased in the not-exerting-control condition (1-sample $t$ test against $\left.0: t_{(24)}=-2.29, p<0.05, d=0.93\right)$, replicating previous findings (Avenanti et al., 2005, 2006). In contrast, MEP amplitude increased in the exerting-control condition (1-sample $t$ test against $\left.0: t_{(24)}=2.63, p<0.05, d=1.07\right)$.

Correlations were computed between the average rating of each subscale of the IRI and the percentage of change in MEP amplitude, averaged for the exerting-control and not-exertingcontrol condition separately. A negative relationship was found between PT and MEP amplitude in the not-exerting-control condition $(r=-0.60, p<0.01$; Fig. $3 A)$. In other words, participants who were more likely to cognitively infer others' states showed a stronger inhibition at the motor level. Furthermore, a positive relationship was found between $\mathrm{PD}$ and MEP amplitude in the exerting-control condition ( $r=0.53, p<0.01$; Fig. $3 B)$, meaning that participants who were more likely to feel distressed about seeing someone else suffering showed a stronger motor facilitation. No other correlations were found between subscales of the IRI and the change in MEP amplitude in the exerting or notexerting-control condition (all $p>0.05$ ).

\section{Discussion}

In this TMS experiment, we investigated whether action tendencies evoked by painful stimuli delivered to the hand of a human model depend on the ability to exert control over the observed hand. Subjects observed the hand of another person receiving painful stimulation after being imitated by this hand or not. During the pain perception phase, TMS-induced MEPs were measured in the right dominant hand of participants. Consistent with previous results, we showed that, when participants did not exert control over the hand that received painful stimulation (i.e., incongruent movements), decreased CSE was found during pain observation. In contrast, when participants exerted control over the hand that received painful stimulation (i.e., congruent movements), increased CSE was observed.

It has been shown repeatedly that observing others in pain generates not only affective responses, but also sensory-motor responses in the observer (Keysers et al., 2010; Lamm et al., 2011). Indeed, several TMS studies exploring reactions to perceiving 

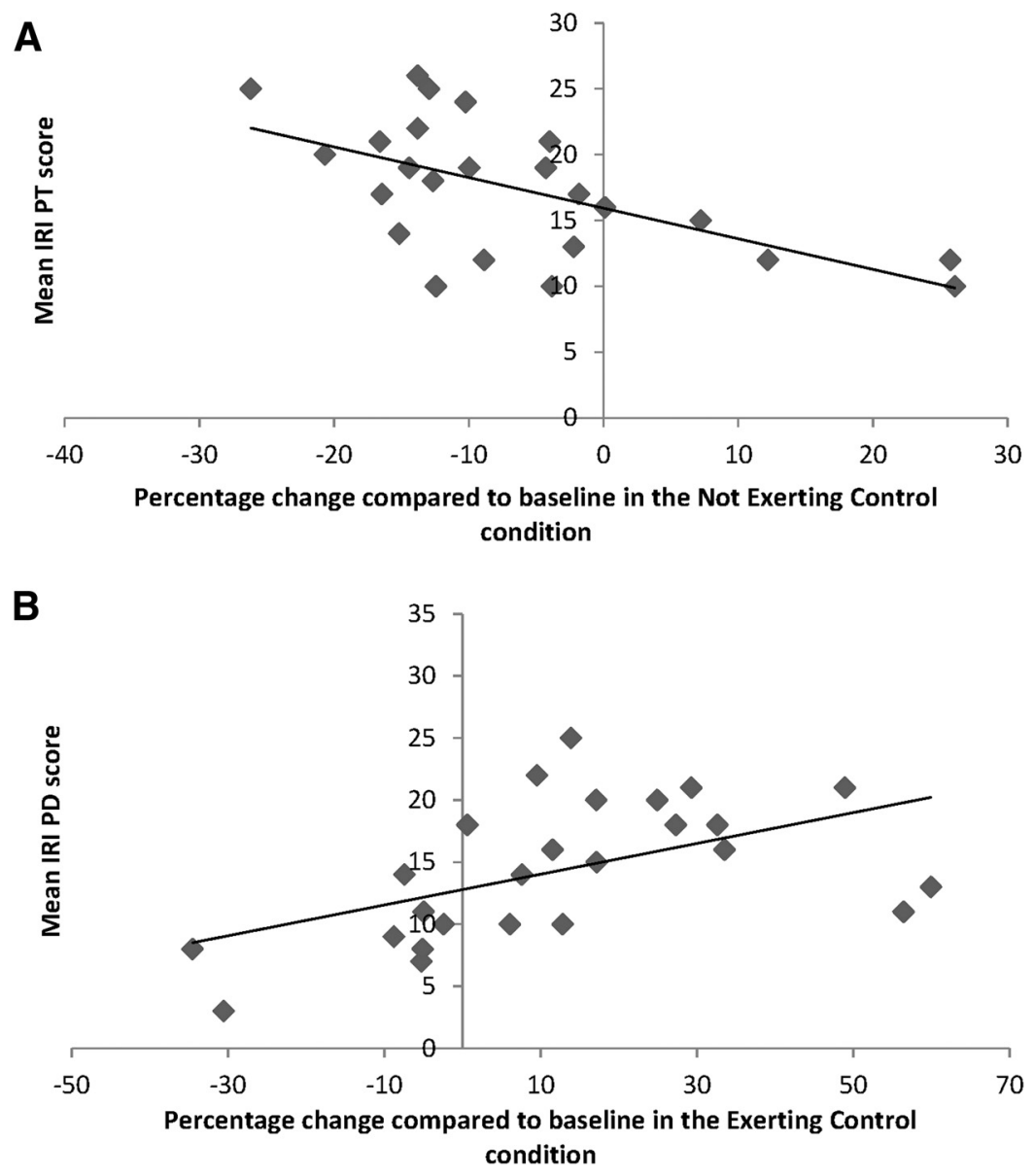

Figure 3. A, Correlation between mean IRI PT score and mean CSE in the not-exerting-control condition. B, Correlation between mean IRI PD score and mean CSE in the exerting-control condition.

pain in others evidenced decreased excitability in the motor system of the observer. This decrease has been shown to be specific to the body part that was hurt in others and to correlate with the pain intensity as estimated by the observer (Avenanti et al., 2005, 2006; Minio-Paluello et al., 2006). It has been argued that this inhibition reflects a freezing response that is similar to the reaction observed when actually experiencing pain (Le Pera et al., 2001; e.g., Farina et al., 2003; Urban et al., 2004). Other research, however, has shown that high levels of personal involvement are associated with reduced motor inhibition during pain observation (Avenanti et al., 2009). Moreover, Fitzgibbon et al. (2012) have shown that pain synesthetes show a significant increase of CSE when observing someone else in pain. The present study accounts for these discrepancies by showing that action tendencies are modulated by the level of control that participants exerted over the hand that received painful stimulation.

Several TMS studies have shown that increased CSE might reflect anticipatory changes to perception of negative emotional cues (Oliveri et al., 2003; Koganemaru et al., 2012; Borgomaneri et al., 2013). Furthermore, it has been shown that the motor system implements anticipatory simulations of expected actions (Kilner et al., 2004; Borroni et al., 2005; Urgesi et al., 2010; Avenanti et al., 2013). We assume that the facilitation of CSE observed in the exerting-control condition reflects planning of an avoidance reaction to the observed pain. In particular, this increased CSE might reflect an unspecific muscle tension halting ongoing behavior to prepare for a potential avoidance response.
Such an avoidance response is only adaptive when the agent has the possibility to escape the painful stimulation. Previous studies examining CSE when experiencing pain oneself used methods (e.g., saline injection, electrical stimulation) that prevent preparation of appropriate reactions to avoid pain (Le Pera et al., 2001; Urban et al., 2004). In such situations in which it is already too late to stop or avoid the painful stimulation, an anesthetic motor inhibition is the most adaptive response. By definition, situations in which participants passively observe pain to the hand of a human model preclude an active avoidance response, so an anesthetic motor inhibition is displayed. In a previous study, De Coster et al. (2013) showed a reduced sense of agency when the hand that receives pain does not imitate the finger movements executed by participants. In this sense, the not-exerting-control condition is similar to observing others in pain without any possibility to prepare an avoidance reaction to this pain. In support of this view and consistent with previous research (Avenanti et al., 2009; Minio-Paluello et al., 2009; Avenanti et al., 2010), a correlation was found in our study between this inhibitory effect and the individual ratings of perspective taking, a cognitive marker of empathy, in the not-exerting-control condition. It seems that, the more a participant feels able to cognitively change his/her perspective to adopt others' point of view, the more he/ she experiences motor inhibition during pain observation. Interestingly, Shamay-Tsoory et al. (2009) suggested that perspective taking is closely related to the Theory of Mind abilities and the awareness that others' states are different from one's own. In contrast, being imitated provides participants with a feeling of control over the model hand due to an increased self-other overlap (De Coster et al., 2013). The more participants are distressed about seeing the hand that they can control, the more they show activation in this hand. This correlation is consistent with the results of Borgomaneri et al. (2013), who found that interindividual differences in personal distress were positively correlated with an increased CSE. Interestingly, facilitory CSE responses when viewing negative stimuli seem to be muscle unspecific (Borgomaneri et al., 2013). This raises the possibility that facilitation of CSE might be part of a more generalized preparatory response toward negative situations, especially because Borgomaneri et al. (2013) indicated the very early nature of these facilitory responses. This might indicate that the first response to a threatening situation is a complete and unspecific muscle tension that serves the role of stopping ongoing behavior and preparing avoidance. Unfortunately, we were not able to test this hypothesis because the data from the only other muscle we measured (the BR muscle) were not reliable.

Consistent with previous results (De Coster et al., 2013), we showed that behavioral self-reports of pain intensity were 
higher in the exerting-control condition compared with the not-exerting-control condition. Furthermore, in this previous study, both other-oriented and self-oriented feelings were rated higher in the exerting-control condition, reflecting concern and personal distress, respectively (Batson et al., 1987). In addition, we found that not only was agency/control higher in the imitation condition, but this condition elicited higher body ownership as well. In particular, we demonstrated that exerting control induced a rubber hand illusion (RHI) indicating enhanced body ownership (De Coster et al., 2013). It is therefore an open question whether the effect of exerting control influenced the action tendency directly or if this effect is mediated by increased body ownership. In any case, ours is the first experimental study showing increased CSE in a situation in which self-other overlap is high. Although it has been shown using fMRI that threatening a rubber hand that feels as if it is your own hand increases brain activity in pain-related and motor-related areas (Ehrsson et al., 2007), the specific nature of the motor response (inhibition or facilitation) cannot be investigated with fMRI. Interestingly, other research (Schütz-Bosbach et al., 2006; Schütz-Bosbach et al., 2009) has shown that, when applying motor TMS in an RHI paradigm (without observation of noxious stimulation), differential modulation of the FDI was present as well. Although asynchronous stimulation (no RHI) led to increased MEP amplitude and reduced cortical silent period duration when observing index finger movements, synchronous stimulation (RHI) led to the opposite pattern. These results confirm the idea that self-other overlap can modulate CSE, reflecting appropriate responses to the observed stimuli.

In addition, our study provides the first systematic evidence that CSE changes induced by pain observation are mediated by the merging of self- other representations. Although it has been widely accepted that the inhibitory effect is due to an embodiment of the observed pain, this has never been demonstrated systematically. With the present paradigm, we have demonstrated that CSE effects in the exerting-control condition are qualitatively different from those in the not-exertingcontrol condition. Our study thus suggests that increasing self-other overlap (due to being imitated in the exerting condition) leads to a facilitation of MEPs when observing pain and that this facilitation is higher for people who are more strongly affected by other's distress. Therefore, these results indicate that being imitated has a strong influence both on emotional reactions, such as empathy for pain, and bodily reactions in the observer. Enhancing self-other overlap by being imitated thus provides a novel and original paradigm for investigating pathological populations, such as autism or schizophrenic individuals, who show altered emotional reactions that are related to deficiencies in self-other representations (e.g., autism, schizophrenia).

In sum, our results indicate that whether we exert control over an observed body part or not determines the nature of the CSE changes consecutive to perceiving pain in others. Whereas having no control leads to motor inhibition when observing someone in pain, exerting control leads to motor facilitation. We argue that this increase in CSE response reflects the tendency to prepare for avoidance of the painful stimulation. In contrast, having no control over the hand elicits an anesthetic response, as evidenced by motor inhibition.

\section{References}

Avenanti A, Bueti D, Galati G, Aglioti SM (2005) Transcranial magnetic stimulation highlights the sensorimotor side of empathy for pain. Nat Neurosci 8:955-960. CrossRef Medline

Avenanti A, Minio-Paluello I, Minio Paluello I, Bufalari I, Aglioti SM (2006) Stimulus-driven modulation of motor-evoked potentials during observation of others' pain. Neuroimage 32:316-324. CrossRef Medline

Avenanti A, Minio-Paluello I, Bufalari I, Aglioti SM (2009) The pain of a model in the personality of an onlooker: Influence of state-reactivity and personality traits on embodied empathy for pain. Neuroimage 44:275283. CrossRef Medline

Avenanti A, Sirigu A, Aglioti SM (2010) Racial bias reduces empathic sensorimotor resonance with other-race pain. Curr Biol 20:1018-1022. CrossRef Medline

Avenanti A, Annella L, Candidi M, Urgesi C, Aglioti SM (2013) Compensatory plasticity in the action observation network: virtual lesions of STS enhance anticipatory simulation of seen actions. Cereb Cortex 23:570580. CrossRef Medline

Batson CD, Fultz J, Schoenrade PA (1987) Distress and empathy-two qualitatively distinct vicarious emotions with different motivational consequences. Journal of Personality 55:19-39. CrossRef Medline

Borgomaneri S, Gazzola V, Avenanti A (2013) Temporal dynamics of motor cortex excitability during perception of natural emotional scenes. Soc Cogn Affect Neurosci. Advance online publication. Retrieved Sep. 12, 2013. doi:10.1093/scan/nst139. CrossRef Medline

Borroni P, Montagna M, Cerri G, Baldissera F (2005) Cyclic time course of motor excitability modulation during the observation of a cyclic hand movement. Brain Res 1065:115-124. CrossRef Medline

Davis MH (1980) A multidimensional approach to individual differences in empathy. JSAS Catalog of Selected Documents in Psychology 10:85.

De Coster L, Verschuere B, Goubert L, Tsakiris M, Brass M (2013) I suffer more from your pain when you act like me: Being imitated enhances affective responses to seeing someone else in pain. Cogn Affect Behav Neurosci 13:519-532. CrossRef Medline

De Corte K, Buysse A, Verhofstadt LL, Roeyers H, Ponnet K, Davis MH (2007) Measuring empathic tendencies: reliability and validity of the Dutch version of the Interpersonal Reactivity Index. Psychologica Belgica 47:235-260.

Ehrsson HH, Wiech K, Weiskopf N, Dolan RJ, Passingham RE (2007) Threatening a rubber hand that you feel is yours elicits a cortical anxiety response. Proc Natl Acad Sci U S A 104:9828-9833. CrossRef Medline

Farina S, Tinazzi M, Le Pera D, Valeriani M (2003) Pain-related modulation of the human motor cortex. Neurol Res 25:130-142. CrossRef Medline

Fitzgibbon BM, Enticott PG, Bradshaw JL, Giummarra MJ, Chou M, Georgiou-Karistianis N, Fitzgerald PB (2012) Enhanced corticospinal response to observed pain in pain synesthetes. Cogn Affect Behav Neurosci 12:406-418. CrossRef Medline

Keysers C, Kaas JH, Gazzola V (2010) Somatosensation in social perception. Nat Rev Neurosci 11:417-428. CrossRef Medline

Kilner JM, Vargas C, Duval S, Blakemore SJ, Sirigu A (2004) Motor activation prior to observation of a predicted movement. Nat Neurosci 7:12991301. CrossRef Medline

Koganemaru S, Domen K, Fukuyama H, Mima T (2012) Negative emotion can enhance human motor cortical plasticity. Eur J Neurosci 35:16371645. CrossRef Medline

Lamm C, Decety J, Singer T (2011) Meta-analytic evidence for common and distinct neural networks associated with directly experienced pain and empathy for pain. Neuroimage 54:2492-2502. CrossRef Medline

Le Pera D, Graven-Nielsen T, Valeriani M, Oliviero A, Di Lazzaro V, Tonali PA, Arendt-Nielsen L (2001) Inhibition of motor system excitability at cortical and spinal level by tonic muscle pain. Clin Neurophysiol 112: 1633-1641. CrossRef Medline

Minio-Paluello I, Avenanti A, Aglioti SM (2006) Left hemisphere dominance in reading the sensory qualities of others' pain? Soc Neurosci 1:320-333. CrossRef Medline

Minio-Paluello I, Baron-Cohen S, Avenanti A, Walsh V, Aglioti SM (2009) Absence of embodied empathy during pain observation in Asperger syndrome. Biol Psychiatry 65:55-62. CrossRef Medline

Oliveri M, Babiloni C, Filippi MM, Caltagirone C, Babiloni F, Cicinelli P, Traversa R, Palmieri MG, Rossini PM (2003) Influence of the supplementary motor area on primary motor cortex excitability during move- 
ments triggered by neutral or emotionally unpleasant visual cues. Exp Brain Res 149:214-221. Medline

Rossi S, Hallett M, Rossini PM, Pascual-Leone A, Pascual-Leone A (2009) Safety, ethical considerations, and application guidelines for the use of transcranial magnetic stimulation in clinical practice and research. Clin Neurophysiol 120:2008-2039. CrossRef Medline

Schütz-Bosbach S, Mancini B, Aglioti SM, Haggard P (2006) Self and other in the human motor system. Curr Biol 16:1830-1834. CrossRef Medline

Schütz-Bosbach S, Avenanti A, Aglioti SM, Haggard P (2009) Don’t do it! Cortical inhibition of self-attribution during action observation. J Cogn Neurosci 21:1215-1227. CrossRef Medline

Shamay-Tsoory SG, Aharon-Peretz J, Perry D (2009) Two systems for empathy: a double dissociation between emotional and cognitive empathy in inferior frontal gyrus versus ventromedial prefrontal lesions. Brain 132: 617-627. CrossRef Medline

Singer T, Seymour B, O’Doherty J, Kaube H, Dolan RJ, Frith CD (2004) Empathy for pain involves the affective but not sensory components of pain. Science 303:1157-1162. CrossRef Medline

Urban PP, Solinski M, Best C, Rolke R, Hopf HC, Dieterich M (2004) Different short-term modulation of cortical motor output to distal and proximal upper-limb muscles during painful sensory nerve stimulation. Muscle Nerve 29:663-669. CrossRef Medline

Urgesi C, Maieron M, Avenanti A, Tidoni E, Fabbro F, Aglioti SM (2010) Simulating the future of actions in the human corticospinal system. Cereb Cortex 20:2511-2521. CrossRef Medline 